\title{
Publisher's Note: Role of the range of the interactions in thermal conduction
}

[Phys. Rev. E 94, 042117 (2016)]

\author{
Carlos Olivares and Celia Anteneodo
}

(Q) (Received 8 April 2019; published 17 April 2019)

DOI: 10.1103/PhysRevE.99.049901

This paper was published online on 17 October 2016 with incorrect page number in Ref. [29]. Reference [29] should read as "Y. Li, N. Li, and B. Li, Eur. Phys. J. B 88, 182 (2015)." The reference has been corrected online as of 8 April 2019. The reference is incorrect in the print version of the journal. 